attack of pleurisy, which was probably in no way connected with the disease (glanders) from which the horse had previously suffered. Acute pleurisy in the horse is sometimes caused by glanders bacilli, but the experiments with the guinea-pigs prove that this case was certainly not of that nature.

Reviewing the whole history of the case, the observations would appear to show that this horse, although showing external symptoms of farcy, became quite cured (or recovered). Afterwards, when inoculated with virulent glanders bacilli, it again contracted the disease, and it had again recovered before it died from another cause. It would be dangerous to infer from this one case that mallein exerts a curative influence on glanders, though that is a perfectly legitimate interpretation of the results. It ought to be pointed out, however, that the quantity of mallein used was very large, and that the period over which its administration was spread was a long one. The case, therefore, does not lend much support to the view that I cc. of mallein used for the purpose of diagnosis may suffice to cure an attack of glanders that otherwise would have developed until it caused death.

\title{
TUBERCULOSIS OF THE SHEEPx
}

By the same.

THE comparative exemption of the sheep from tuberculosis is a remarkable fact, in view of the great prevalence of the disease among cattle, and its far from infrequent occurrence among pigs, horses, dogs, and poultry. There would, of course, be nothing remarkable in this exemption, any more than there is in the exemption of sheep from contagious pleuro-pneumonia, if tuberculosis were not a disease communicable to the ovine species; but the sheep has no absolute immunity against tuberculosis, for occasional natural cases of the disease among these animals are met with, and sheep may be experimentally infected without any difficulty either by inoculation or feeding.

In evidence of the rarity of the disease among British sheep, it may be stated that there has not yet been placed on record one indubitable case in this country. On many occasions I have had brought under my notice what were alleged to be examples of tuberculosis in the sheep; but when the affected parts were forthcoming for examination the disease always turned out to be of some other nature, most frequently nodular lesions caused by worm parasites. On account of its rarity, considerable interest therefore attaches to the following case.

In the month of December I898, part of the side of a sheep seized in a slaughter-house was sent to me by $\mathrm{Mr}$ W. F. Shaw, F.R.C.V.S. On the inner aspect of the chest wall and on the upper surface of the breast-bone there were some clusters of nodules very like the so-called "grapes" which are so frequently found in tuberculosis of cattle. All these nodules had a fibrous capsule, enclosing contents which were completely caseous and partially calcified, except in the case of one at the hinder end of the breast- 
bone, which contained soft caseo-purulent material. The appearance of these lesions naturally suggested that they were tuberculous, and, indeed, it was for verification of that diagnosis that $\mathrm{Mr}$ Shaw had sent the parts. A microscopic examination did not reveal any tubercle bacilli in the diseased parts; but in spite of this it was still suspected that the disease was tuberculosis, and with a view to obtaining positive evidence to that effect a little particle of the soft material from the nodule near the end of the breast-bone was introduced under the skin of each of two rabbits. This experiment was performed on Ioth December, I 898. One of the rabbits died on I 9 th A pril, and the other on I3th May, I 899, and the lesions found in them were almost identical in the two cases. There was some disease at the place where the matter had been introduced under the skin, the nearest lymphatic glands were enlarged and caseating, and numerous tubercles were present in the lungs and kidneys. Tubercles were also present in the liver of one of the rabbits, but they were absent from the. spleen in both. All doubt as to the nature of the disease in the sheep was set at rest by finding that tubercle bacilli were present in the lesions in the rabbits.

From the rabbit which died on I 3 th May another was inoculated, using for the purpose a particle of matter from the enlarged and caseating lymphatic gland in the axilla. This third rabbit died from tuberculosis on 2 oth August, and from a tubercle in one of its lungs a fourth was inoculated. It died on Ioth December, with tuberculous disease of the lymphatic glands, lungs, and kidneys. From the lungs of this rabbit tubercle bacilli were obtained in pure culture.

It would have greatly enhanced the interest of this case if the history of the sheep had been obtainable, but nothing could be learned with regard to that, and it is therefore impossible to say how the disease was contracted. As has already been stated, this is the first undoubted case of tuberculosis in the sheep that has been recorded in this country, but every year a number of such cases are detected in the large German slaughter-houses. Probably the more frequent occurrence of the disease among German sheep is ascribable to the greater frequency with which sheep in some parts of the Continent are housed during the winter months, for there can be little doubt that it is in large measure the open-air existence of the sheep which secures for it an almost complete exemption from tuberculosis in this country. The beneficial effect of an open-air mode of life in this respect is probably not so much due to its invigorating influence, as to the obstacles which it places in the way of infection from animal to animal. 